\title{
Depression and its treatment - a mini review
}

\begin{abstract}
Major depressive disorder (MDD) is one of the most common mental health conditions in the United States. Depressed mood for at least two-week period could be diagnosed as MDD. A variety of pathological and neurotransmitter changes could result in the development of the MDD. Combination of non-pharmacological and pharmacological therapies could be utilized to treat the depression. The antidepressant use could result in their respective side effects. Therefore, the choice of medication needs to be tailored keeping in consideration the individual characteristics and safety profile.
\end{abstract}

Keywords: major depressive disorder, antidepressants, SSRI, SNRI, TCA
Volume 9 Issue 6 - 2018

\author{
Ansari lqra,' Jabbar Qammar, ${ }^{2}$ Shah A Asim 3,4 \\ 'Assistant Physician, Metro Cardiovascular Clinic St. Louis, USA \\ ${ }^{2}$ Graduate from Jinnah Sindh Medical University, Pakistan \\ ${ }^{3}$ Department of Psychiatry and Behavioral Sciences, Baylor \\ College of Medicine, USA \\ ${ }^{4}$ Chief of Psychiatry, Ben Taub Hospital, Harris Health System, \\ USA
}

Correspondence: Iqra Ansari MD, Graduate of Jinnah Sindh Medical University, Karachi, Pakistan and 780 Pifer Road Houston, Texas, USA, Tel + |4697851217,

Email Ansari_iqva@yahoo.com

Received: July 24, 2018 | Published: November 07, 2018

\section{Introduction}

Major depressive disorder (MDD) is diagnosed when depressed mood or loss of pleasure symptoms remains for at least a twoweek period. Patient might complain of feeling sad, hopeless, reduced energy levels, weight changes, sleep disturbances, fatigue, disproportionate guilt, and lack of concentration, suicidal ideation with or without particular plans. In the USA, $6.7 \%$ of all United States (US) adults at least had one episode of major depression. The prevalence of major depressive episode was higher in females $(8.5 \%)$ than the males $(4.8 \%){ }^{1}$

\section{Pathophysiology}

A number of hypotheses are associated with the development of MDD. Genetic and psychosocial stress has shown to affect the disease process. Hypothalamic pituitary adrenal (HPA) axis changes, monoamine deficiencies, specific brain region dysfunction, reduced levels and action of Gamma-aminobutyric acid (GABA), affected glutamate regulation, neurotoxic processes and circadian rhythm disturbances are few of the proposed mechanisms that leads to the development of MDD. ${ }^{2}$ Medical conditions which could lead to depressive symptoms include autoimmune disorders, neurologic conditions (stroke, seizure disorder, brain injury), dementia, malignancies, endocrine disorders (hypercalcemia, hyperthyroidism, hypothyroidism, hyperparathyroidism) and infectious disorders (HIV, hepatitis). Substances and medications which cause depressive symptoms include oral contraceptives, sedatives, thiazide, propranolol, thiazide, alcohol and antiparkinsonian medications. ${ }^{3}$

\section{Diagnosis}

The MDD is diagnosed on clinical evaluation or by conducting structured diagnostic interview (SDI). Dexamethasone suppression test is a laboratory method to diagnose depression. Patient Health Questionnaire 9 (PHQ-9) is an easy to use, self-administered diagnostic tool. Some emerging tools in the diagnostic sciences for depression are genomic, proteomic and metabolomic methods. Various psychiatric conditions affect different biological pathways. Pro-inflammatory and anti-inflammatory cytokines have been associated with the MDD. ${ }^{4}$ IL- $1 \alpha$, IL-1 $\beta$, IL-2, IL-6, IL-8 and Interferon gamma are few of the cytokines which have been found elevated in patients with MDD when compared to control subjects. ${ }^{5}$ Other than the MDD, various form of depression includes bipolar depression, mixed depression, atypical depression, melancholic depression, minor depressive disorder, recurrent brief depressive disorder, seasonal affective disorder and dysthymic disorder. ${ }^{6}$

\section{Treatment}

Patients with mild or moderate depression could benefit from exercise therapy. Cognitive behavioral therapy (CBT) is another recommendation for mild or moderate depression in adults. The efficacy of CBT has been comparable to other interpersonal psychotherapies or other types of psychotherapies. Combination of interpersonal therapy and pharmacological treatment together could be more efficacious for severe depression. However, so far, the clinical trials have proven two modalities to be most efficacious for the treatment of depression, pharmacological therapy (the antidepressants) and the $\mathrm{CBT}^{7}$

\section{Pharmacological treatment}

There are various antidepressants that are currently used. The list includes the tricyclic antidepressants (TCAs), Selective serotonin reuptake inhibitors (SSRIs), Selective serotonin norepinephrine reuptake inhibitors (SNRIs), Serotonin and norepinephrine receptor antagonists, dopamine reuptake inhibitors, monoamine oxidase inhibitors (MAOIs) and others. Mood stabilizers (Lithium salts and epileptics) have also been used as an adjunct therapy (Table 1).

Table I Pharmacological treatment of depression

Selective serotonin reuptake inhibitor (SSRIS).

Serotonin Norepinephrine Reuptake inhibitors (SNRIs).

Tricyclic antidepressants (TCAs).

Monoamine oxidase inhibitors (MAOls).

Other antidepressants (Bupropion, Mirtazapine, Trazadone).

Antipsychotic (Aripiprazole, Quetiapine). 


\section{Selective Serotonin Reuptake Inhibitors (SSRIs)}

In 1988, Fluoxetine was introduced in the USA. SSRIs have the comparable efficacy to the TCAs but remarkably lesser side effects. Paroxetine, Fluvoxamine, Citalopram, Escitalopram and Sertraline are the common SSRIs used. SSRIs increase the concentration of serotonin primarily in the hippocampus but it also modulates the level of other neurotransmitters such as norepinephrine and dopamine. SSRI when discontinued after a longer period of use could result in SSRI discontinuation syndrome. In order to avoid it, gradual tapering down of the dose should be accomplished rather than sudden termination. ${ }^{8}$ The common side effects reported with the use of SSRIs are sexual dysfunction, sweating and dreams. Other less common adverse effects reported are dry mouth, insomnia, nausea, fatigue, weight gain, dizziness and tremors. Most SSRIs are metabolized by cytochrome P450 2D6. Safety profile and side effects varies among individuals depending on the genetic polymorphism, personality dimensions, dosage timing, association with food intake and smoking status. ${ }^{9}$ Most SSRI's like Fluoxetine have similar efficacy but safer side-effect profile than TCAs. It is safer if overdosed and less discontinued. ${ }^{10}$ Paroxetine is a potent antidepressant which also is used in the treatment of other psychiatric conditions such as generalized anxiety disorder. Its use is contraindicated in pregnancy due to congenital birth defects i.e. Ventral septal defects. Like other SSRI, it increases the risk for suicidality. Paroxetine has also been associated with increased risk of anti cholinergic side effects, and can cause confusion in elderly. Use of other SSRIs is preferred over Paroxetine in pregnancy and in elderly. ${ }^{11}$

Citalopram has found to be more efficacious than Paroxetine and Reboxetine but less efficacious than Escitalopram, ${ }^{12}$ although dosing was an issue in this study. Citalopram has a warning by FDA about prolongation of QT interval, in higher doses, especially over $40 \mathrm{mg}$. Patients with metabolic disturbances and pre-existing heart conditions are more prone to QT prolongation. ${ }^{13}$ Sertraline is comparable to other SSRIs in terms of safety profile and adverse events. However, more gastrointestinal events such as abdominal pain and diarrhea are reported with the use of sertraline in comparison to other SSRIs. ${ }^{14}$ Comprehension of individual's characteristics of each SSRI is crucial in order to choose one over another. Half-life of each SSRI could affect the severity of withdrawal symptoms. Paroxetine due to its short life is more prone to cause withdrawal symptoms, while fluoxetine due to its long half life has usually no withdrawal. Each individual responds differently to the SSRIs, therefore predicting the most effective drug for that person is a difficult task. Nonetheless, evaluation of personal characteristics is necessary to choose the right medication. The pharmacokinetics, renal and hepatic functions and side effects profile are few major factors that need to be considered.

\section{Serotonin Norepinephrine Reuptake inhibitors (SNRIs)}

According to some studies, SNRIs such as Venlafaxine and Duloxetine in appropriate higher doses are superior to SSRIs for severe depression treatment. ${ }^{15}$ In order to prevent recurrence of major depressive disorder, it is crucial to maintain remission. Agents with dual uptake inhibitions such as SNRIs are being considered as an initial treatment because of their favorable outcomes in maintaining remission. ${ }^{16}$ Genetic variations in cytochrome P450 (CYP) 2D6 activity affects Venlafaxine levels as well, decreased activity of 2D6 results in higher adverse events. Venlafaxine use has been associated with transient and sustained, dose related elevation in diastolic blood pressure. ${ }^{17}$ Venlafaxine has also been associated with severe withdrawal symptoms that may appear within hours of medication cessation. The motor and coordination skills of an individual are affected. ${ }^{18}$ Venlafaxine is an attractive option for treatment of depression in geriatric population, it is a suitable option for conditions such as stroke, anxiety and neuropathic pain which are some frequent comorbid conditions in geriatric population. ${ }^{19}$ Nausea, dry mouth, constipation, appetite change, lack of energy, sleepiness and sweating are few common side effects which are similar to the other antidepressants. Duloxetine has no effect on EKG; it increases heart rate and has very slight effect on weight or blood pressure. ${ }^{20}$

\section{Tricyclic antidepressants (TCAs)}

The newer antidepressants are equally efficacious to the TCAs for the treatment of mild and moderate depression. However, like SNRIs, TCAs are found to be more efficacious for the treatment of severe depression. Discontinuation rate due to side effects is higher with TCAs than the newer antidepressants. Interaction with other drugs such as SSRIs could cause increased tricyclic side effects. Overdose of TCAs could be lethal. Despite the advent of newer antidepressants and their efficacies, TCAs still hold an important role in management of severe depression. ${ }^{21}$ The increase rate of drug discontinuation is because of increased TCA side effects such as dry mouth, dizziness, fatigue and drowsiness. ${ }^{22}$ Statistically significant fall in systolic and diastolic blood pressure has been associated with the use of clomipramine. ${ }^{23}$ Amitriptyline is choice of drug for inpatient treatment of depression. ${ }^{24}$

\section{Monoamine oxidase inhibitors (MAOIs)}

MAOIs are considered third or fourth line drugs for the treatment of depression because of the side effect profile and need for food restriction. MAOIs are employed for the treatment of atypical depression. Newer transdermal MAOIs have lesser need for dietary restrictions and has fewer sexual and metabolic adverse events. ${ }^{25}$ MAOIs interaction with other antidepressants could result in serotonin syndrome. MAOIs if taken with any other adrenergic medication can cause hypertensive crisis. Selegiline transdermal system (STS) was formulated considering the pharmacokinetic properties which would inhibit MAO-A and MAO-B in the central nervous system but not the liver and intestine. STS result in higher level of serum concentration in comparison to the oral medication as it is not subjected to the first pass metabolism. ${ }^{26}$

\section{Other antidepressants}

Bupropion inhibits reuptake of norepinephrine and dopamine as well as potentiate the effect of other antidepressants such as SSRIs. It was approved in 1989 by FDA for the treatment for major depression. However, in 1997 it was also allowed to be used as an aid for smoking cessation. Bupropion is used in Adult Attention deficit hyperactive disorder and weight loss as an off-label management. ${ }^{27}$ Mirtazapine has been considered for the treatment of elderly depression. Fatigue, weight gain and sedation are the main side effects of mirtazapine treatment. Mirtazapine causes less cholinergic and $\alpha 1$ adrenergic side effects similar to SSRIS and SNRIs. It also causes less nauseas and sexual problems due to its 5 HT2 and 5HT3 receptor blockade. ${ }^{28}$ Mirtazapine has shown to increase gastric tone. Animal studies have shown improved gastric emptying. ${ }^{29}$ Trazadone is a potent antidepressant with beneficial effect on sleep cycle. It is used for the treatment of depression as a monotherapy as well as combination therapy. Trazadone has limited anticholinergic activity. However, in patient with pre-existing heart diseases such as elderlies, it should be used with cautious. ${ }^{30}$ 


\section{Antipsychotic}

Atypical antipsychotics can be use an additive therapy for the treatment of major depression. Food and drug administration (FDA) has so far approved aripiprazole and quetiapine as an adjunctive treatment. When administering antipsychotics, there side effects such as weight gain, extra-pyramidal symptoms and hyperglycemia needs to be monitored..$^{31}$

\section{Alternate and newer therapies}

Some of the less conventional yet alternate available therapies are light therapy, electroconvulsive therapy, magnetic stimulation, vagus nerve stimulation and deep brain stimulation. ${ }^{32}$ All have questionable efficacy except ECT, and in very resistant cases DBS.

\section{Acknowledgement}

None.

\section{Conflict of interest}

The author declares that there is no conflict of interest.

\section{References}

1. National institute of mental health. Major depression. 2017.

2. Hasler G. Pathophysiology of depression: do we have any solid evidence of interest to clinicians? World Psychiatry. 2010;9(3):155-161.

3. Soleimani L, Lapidus KA, Losifescu DV. Diagnosis and treatment of major depressive disorder. Neurol Clin. 2011;29(1):177-193.

4. Smith KM, Renshaw PF, Bilello J. The diagnosis of depression: current and emerging methods. Compr Psychiatry. 2013;54(1):1-6.

5. Simon NM, McNamara K, Chow $\mathrm{CW}$, et al. A detailed examination of cytokine abnormalities in Major Depressive Disorder. Eur Neuropsychopharmacol. 2008;18(3):230-233.

6. Benazzi F. Various forms of depression. Dialogues Clin Neurosci. 2006;8(2):151-161.

7. Park SC, Oh HS, Oh DH, et al. Evidence-based, non-pharmacological treatment guideline for depression in Korea. J Korean Med Sci. 2014;29(1):12-22.

8. Renoir T. Selective serotonin reuptake inhibitor antidepressant treatment discontinuation syndrome: a review of the clinical evidence and the possible mechanisms involved. Front Pharmacol. 2013;16(4):45.

9. Cascade E, Kalali AH, Kennedy SH. Real-World Data on SSRI Antidepressant Side Effects. Psychiatry (Edgmont). 2009;6(2):16-18.

10. Rossi A, Barraco A, Donda P. Fluoxetine: a review on evidence-based medicine. Ann Gen Hosp Psychiatry. 2004;3(1):2.

11. Nevels RM, Gontkovsky ST, Williams BE. Paroxetine-The Antidepressant from Hell? Probably Not, But Caution Required. Psychopharmacol Bull. 2016;46(1):77-104.

12. Cipriani A, Purgato M, Furukawa TA, et al. Citalopram versus other anti-depressive agents for depression. Cochrane Database Syst Rev. 2012;(7):CD006534
13. Cooke MJ, Waring WS. Citalopram and cardiac toxicity. Eur J Clin Pharmacol. 2013;69(4):755-760.

14. Meijer WE, Heerdink ER, Van Eijk JT, et al. Adverse events in users of sertraline: results from an observational study in psychiatric practice in The Netherlands. Pharmacoepidemiol Drug Saf. 2002;11(8):655-662.

15. Isaac MT. Treating depression with SNRIs: who will benefit most? CNS Spectr. 2008;13(7 Suppl 11):15-21.

16. Zajecka JM, Albano D. SNRIs in the management of acute major depressive disorder. J Clin Psychiatry. 2004;65 Suppl 17:11-18.

17. Thase ME. Effects of venlafaxine on blood pressure: a meta-analysis of original data from 3744 depressed patients. J Clin Psychiatry. 1998;59(10):502-508.

18. Campagne DM. Venlafaxine and serious withdrawal symptoms: warning to drivers. MedGenMed. 2005;7(3):22.

19. Staab JP, Evans DL. Efficacy of venlafaxine in geriatric depression. Depress Anxiety. 2000;12 Suppl 1:63-68.

20. Goldstein DJ. Duloxetine in the treatment of major depressive disorder Neuropsychiatr Dis Treat. 2007;3(2):193-209.

21. Boyce P, Judd F. The place for the tricyclic antidepressants in the treatment of depression. Aust N Z J Psychiatry. 1999;33(3):323-327.

22. Wilson K, Mottram P. A comparison of side effects of selective serotonin reuptake inhibitors and tricyclic antidepressants in older depressed patients: a meta-analysis. Int J Geriatr Psychiatry. 2004;19(8):754-762.

23. Stage KB. Orthostatic side effects of clomipramine and moclobemide during treatment for depression. Nord J Psychiatry. 2005;59(4):298-301.

24. Barbui C, Guaiana G, Hotopf M. Amitriptyline for inpatients and SSRIs for outpatients with depression? Systematic review and meta-regression analysis. Pharmacopsychiatry. 2004;37(3):93-97.

25. Thase ME. MAOIs and depression treatment guidelines. $J$ Clin Psychiatry. 2012;73(7):e24.

26. Culpepper L. Reducing the Burden of Difficult-to-Treat Major Depressive Disorder: Revisiting Monoamine Oxidase Inhibitor Therapy. Prim Care Companion CNS Disord. 2013;15(5): PCC.13r01515.

27. Berigan TR. The Many Uses of Bupropion and Bupropion Sustained Release (SR) in Adults. Prim Care Companion J Clin Psychiatry. 2002;4(1):30-32.

28. Nutt D. Mirtazapine: pharmacology in relation to adverse effects. Acta Psychiatr Scand Suppl. 1997;391:31-37.

29. Yin J, Song J, Lei Y, Prokinetic effects of mirtazapine on gastrointestinal transit. Am J Physiol Gastrointest Liver Physiol. 2014;306(9):G796-801.

30. Fagiolini A, Comandini A, Catena Dell'Osso M. Rediscovering trazodone for the treatment of major depressive disorder. CNS Drugs. 2012;26(12):1033-1049.

31. Wang P, Si T. Use of antipsychotics in the treatment of depressive disorders. Shanghai Arch Psychiatry. 2013;25(3):134-140.

32. Duval F, Lebowitz BD, Macher JP. Treatments in depression. Dialogues Clin Neurosci. 2006;8(2):191-206. 